\title{
Scanning X-ray Microscopy Investigations into the Electron Beam Exposure Mechanism of Hydrogen Silsesquioxane Resists
}

Deirdre L. Olynick, ${ }^{\dagger a}$ Alexei V. Tivanski, ${ }^{*}$ Mary K. Gilles, ${ }^{*}$ Tolek Tyliszczak, ${ }^{\prime}$ Farhad Salmassi, ${ }^{+}$and J. Alexander Liddle

${ }^{\dagger}$ Lawrence Berkeley National Laboratory, Molecular Foundry, Lawrence Berkeley National Laboratory, Chemical Sciences Division

"Lawrence Berkeley National Laboratory, Advanced Light Source

${ }^{+}$Lawrence Berkeley National Laboratory, Center for X-ray Optics

1 Cyclotron Road, Berkeley CA 94720 USA

Kathy Liang, and Stephen R. Leone

Lawrence Berkeley National Laboratory, Chemical Sciences Division, and

University of California, Berkeley, Departments of Chemistry and Physics, Berkeley, CA 94720 USA

\begin{abstract}
Electron-beam exposed hydrogen silsesquioxane cross-linking chemistry is investigated by Scanning Transmission X-ray Microscopy (STXM) and Atomic Force Microscopy (AFM). Using

STXM, a maximum in the chemical contrast is obtained by measuring the X-ray absorption at 534.5

$\mathrm{eV}$, corresponding to the $1 \mathrm{~s} \mathrm{K-edge} \mathrm{transition} \mathrm{in} \mathrm{oxygen.} \mathrm{An} \mathrm{area-dependent} \mathrm{and} \mathrm{dose-dependent}$ chemical conversion is observed for feature sizes between $150 \mathrm{~nm}$ and $10 \mu \mathrm{m}$ and doses between $0.4 \mathrm{mC}$ and $40 \mathrm{mC}$. The activated (cross-linked) regions extend beyond the exposure zones, especially for higher dosed exposures. With AFM, density changes in the latent images (e-beam exposed but undeveloped) are observed, which also display a dependence on exposed area.

Potential mechanisms, involving chemical diffusion outside the exposure zone, are discussed.

a electronic mail: dlolynick@lbl.gov

${ }^{\neq}$Present address: National Institute of Standards and Technology, Gaithersburg, MD
\end{abstract}




\section{Introduction}

Since its initial discovery as a high-resolution negative-tone electron-beam resist, ${ }^{1}$ hydrogen silsesquioxane (HSQ) has become popular for direct-write e-beam, ${ }^{2,3-5}$ extreme ultraviolet (EUV), ${ }^{6}$ and nanoimprint lithographic applications. ${ }^{7,8} \mathrm{HSQ}$ is attractive as a resist for a variety of reasons including demonstrated high-resolution down to $12.5 \mathrm{~nm}$ half-pitch gratings ${ }^{5}$ with a sensitivity comparable to Zeon corporation's ZEP-520 or polymethyl methacralate (PMMA). In addition HSQ exhibits excellent dry etch pattern transfer characteristics, especially for bi-layer applications using oxygen plasma chemistry, ${ }^{5,8,9}$ because it undergoes further curing in that environment. ${ }^{10}$

HSQ was initially studied and marketed by Dow Corning as a spin-on Inter-Layer Dielectric (ILD) material, FOx ${ }^{\circledR}$. In addition to a low dielectric constant, it has excellent planarization and gapfill characteristics. The thermal curing of HSQ from its as-spun state to a form suitable for Si device integration has been studied extensively. ${ }^{11}$ The dielectric constant is sensitive to the final cured structure, which is in turn determined by the ambient bake conditions, temperature cycling, and presence of moisture.

The fully condensed HSQ structure has a formula of $\left(\mathrm{HSiO}_{3 / 2}\right)_{\mathrm{n}} \cdot{ }^{12,13}$ For $\mathrm{n}=8$ the proposed structure is cubic with silicon at the corners and every silicon bound to 3 oxygens and a single hydrogen. The cured structure is described as a network structure, which, under typical curing conditions, leads to an increase in the $\mathrm{Si}-\mathrm{O}$ to $\mathrm{Si}-\mathrm{H}$ bond ratio. ${ }^{11}$ The mechanism leading to dissolution contrast after e-beam exposure is not yet clear. Based on FTIR investigations, Namatsu et. al. ${ }^{1}$ speculated that contrast is achieved in a two step mechanism. Initially $\mathrm{Si}-\mathrm{H}$ bonds are broken to form radical sites. Then, radical sites on neighboring cages become bridged with oxygen in the presence of absorbed water. This is a reasonable assumption; hydroxide is known to be an effective catalyst for 
the oxidation of $\mathrm{Si}-\mathrm{H}$ to $\mathrm{Si}-\mathrm{OH}$ by water, and protons, hydroxide, or traces of water are known to be involved as reactants or catalysts in many polymer reactions. However, this is not the complete picture. Since Namatsu's initial work, ${ }^{1}$ a number of authors have noted a degree of instability and irreproducibility in this material. ${ }^{9,14,15}$ Interestingly it also appears that the e-beam exposure sensitivity of HSQ to e-beam exposure exhibits a dependence on the area exposed. ${ }^{15}$

Recently, scanning transmission x-ray microscopy (STXM) was used to investigate the spatial distribution of the deprotection reaction occurring in a typical chemically amplified resist. ${ }^{16}$ This analysis technique measures the reaction zone with chemical specificity and high spatial resolution $(\sim 35 \mathrm{~nm})$. In this paper, we employ the same STXM techniques in combination with AFM studies of the e-beam exposed latent image to elucidate the details of the chemistry and possible area dependence of the sensitivity that occurs during electron-beam exposure of HSQ. These x-ray chemical imaging studies show that chemical changes occur over micron length scales outside the exposed area and atomic force microscopy investigations indicate that density changes in the exposed film may also play a role. We speculate that diffusion of reactants catalyze changes in both the chemistry and the sensitivity to exposure, both inside and outside the exposed regions.

\section{STXM Background}

The details of the STXM technique as applied to resist studies have been described elsewhere. ${ }^{16}$ Briefly, synchrotron-generated soft x-rays are monochromated and focused to a diffraction-limited spot (in this case $35 \mathrm{~nm}$ FWHM) by a Fresnel zone plate. Images are obtained by measuring the intensity of the transmitted x-rays as the HSQ sample is raster-scanned through the focused beam. Spectra can be recorded at multiple photon energies to interrogate several atomic transitions in the sample. Sharp spectral features are observed close to the absorption edge of specific elements, referred to as "near edge x-ray absorption fine structure."17 The transmitted signal 
is converted to optical density (OD) and is sensitive to the sample thickness, density, and composition according to the following equation:

$$
O D=-\ln \left(\frac{I}{I_{o}}\right)=\sigma t=\mu \rho t
$$

Where $I_{o}$ is the incident photon flux intensity, $\sigma$ the linear absorption coefficient of the film, $t$ the thickness, $\mu$ the mass absorption coefficient and $\rho$ the density. If plotting absorbance, the sign is positive, if plotting transmittance, the sign is negative. If the absorption is too low (sample too thin) the signal may be lost in the noise. If the absorption is too high, the signal can lose quantitative accuracy. Optimally, the OD should be between 0.1 to 3. A sample thickness of $250 \mathrm{~nm}$ was chosen to optimize the absorption at the oxygen edge at values below an OD of 1 . To emphasize the contrast between the e-beam exposed and unexposed regions, where indicated, images are plotted with the natural logarithm of the ratio of the exposed region as I and the unexposed region as $\mathrm{I}_{\mathrm{o}}$, analogous to Eqn. 1, where the changes in the mass absorption coefficient, density, and thickess are probed. This permits a quantitative determination of the empirical change in transmission between the exposed and unexposed regions.

The STXM at beamline 11.0.2 of the Advanced Light Source is used for these experiments. This is an undulator beamline optimized for an energy range from 200 to $1900 \mathrm{eV}$ which covers the O 1s, and Si 1s absorption edges. All experiments reported here are performed at the oxygen absorption edge, although chemical contrast is also observed on the silicon edge. The ambient atmosphere in the STXM used in these experiments is $~ 300$ Torr of He. HSQ is sensitive to exposure to x-rays. Even with very low fluxes and short dwell times, some contrast is always observed between the regions exposed to X-rays for STXM imaging and unexposed regions. To minimize x-ray exposure during image acquisition, images are normally measured at only 1 to 4 
wavelengths. For acquisition of the spectra that requires x-ray exposure at many wavelengths, a defocused beam is used.

\section{HSQ preparation}

As STXM is a transmission technique, samples are prepared on $100 \mathrm{~nm}$ thick silicon nitride membranes. First, a grid with metal alignment markers is prepared on the membranes. These alignment marks provide high transmission contrast and facilitate the location of the HSQ latent images in the STXM. To create the alignment markers, a positive resist is spun on a thin film stack of $12 \mathrm{~nm}$ of Au on $5 \mathrm{~nm} \mathrm{Cr}$ (used as a conductive electroplating base). Exposed and developed samples are electroplated to $250 \mathrm{~nm}$ thickness to create the alignment markers. After removing the photoresist with an oxygen plasma, hydrogen silsesquioxane films are applied. The samples are spun on with $6 \%$ solutions of FOx-15® (available from Dow Corning) or XR-1541 (the prediluted and filtered HSQ product from Dow, which is specifically prepared for e-beam applications) with a thickness of $250 \mathrm{~nm}$.

Studies of latent image e-beam exposure are conducted at an energy of $534.5 \mathrm{eV}$. HSQ films are baked for $5 \mathrm{~min}$ at $170{ }^{\circ} \mathrm{C}$ and patterned using a vector scan $100 \mathrm{keV}$ electron-beam lithography system (Leica VB6HR) with a pattern generator developed at LBNL. ${ }^{18}$ Four exposed feature types are

probed: (1) $10 \mu \mathrm{m}$ squares with $20 \mu \mathrm{m}$ pitch, Dose $(\mathrm{mC})=0.4 * 1.05^{(\mathrm{n}-1)}, \mathrm{n}=1-72 ;$ (2) $1 \mu \mathrm{m} \times 10 \mu \mathrm{m}$ lines with $2 \mu \mathrm{m}$ pitch; Dose $(\mathrm{mC})=0.4 * 1.05^{(\mathrm{n}-1)}, \mathrm{n}=1-100$; (3) $150 \mathrm{~nm} \times 10 \mu \mathrm{m}$ lines with $1 \mu \mathrm{m}$ pitch; Dose $(\mathrm{mC})=0.4 * 1.03^{(\mathrm{n}-1)}, \mathrm{n}=1-150$, (4) $150 \mathrm{~nm} \times 10 \mu \mathrm{m}$ lines with $1 \mu \mathrm{m}$ pitch; Dose $(\mathrm{mC})$ $=0.4 * 1.1^{(\mathrm{n}-1)}, \mathrm{n}=1-40$. The minimum feature size was set to obtain multiple pixels per feature (STXM spatial resolution was $35 \mathrm{~nm}$ ). STXM data acquisition was begun within 1 hour of removing the sample from the e-beam system.

Tapping mode AFM measurements (Digital Instruments 3100, standard silicon tips) of the 
latent images are taken from the samples prepared for STXM and also from $250 \mathrm{~nm}$ thick exposed HSQ films prepared on bare Silicon wafers.

\section{STXM results}

Figure 1 compares NEXAFS data at the oxygen edge from an e-beam exposed area and an unexposed region of the same sample. Spectral features in this energy region arise from the excitation of an oxygen $1 \mathrm{~s}$ electron. The spectrum from the region that has not been e-beam exposed displays a distinct narrow peak at $535.8 \mathrm{eV}$ and a second, broader peak at $539 \mathrm{eV}$. In the spectrum of the e-beam exposed material the first peak is absent, while the second peak appears to have broadened significantly. These subtle changes in the NEXAFS spectra can be used to determine the x-ray energies where the largest contrast occurs between the e-beam exposed and unexposed samples. To obtain an OD difference spectra (Fig. 1, bottom, $\mathrm{OD}_{\text {difference }}=-\ln \left(\mathrm{I}_{\text {exposed }} / \mathrm{I}_{\text {unexposed }}\right)$, where I is the transmitted intensity), latent e-beam images are measured at 159 energies between $520-560 \mathrm{eV}$, at a dwell of $0.5 \mathrm{~ms}$ per pixel with a defocused beam (Fig. 1, bottom). In this case, both spectra are normalized by an $\mathrm{I}_{\mathrm{o}}$ that is taken using a blank $\mathrm{Si}_{3} \mathrm{~N}_{4}$ window.

STXM images of latent e-beam exposures of HSQ (10 $\mu \mathrm{m}$ squares) are shown in Fig. 2 and plotted as $\left|-\ln \left(\mathrm{I}_{\text {exposed }} / \mathrm{I}_{\text {unexposed }}\right)\right|$ (this and subsequent transmitted intensities are not normalized by an $\mathrm{I}_{\mathrm{o}}$ through a blank $\mathrm{Si}_{3} \mathrm{~N}_{4}$ window). Images are obtained at $525 \mathrm{eV}$ (Fig. 2A), $534.2 \mathrm{eV}$ (Fig. 2B), 535.4 eV(Fig. 2C), and $537.2 \mathrm{eV}$ (Fig. 2D). At energies below the oxygen 1s transition (Fig. 2A), no chemical contrast is observed between the exposed and unexposed areas. At $534.2 \mathrm{eV}$ (Fig. 2B), contrast can be seen and the exposed region of the image is less transmissive, represented by lighter features on a darker background ( $\left.\mathrm{I}_{\text {unexposed }}>\mathrm{I}_{\text {exposed }}\right)$, and the counterintuitive light versus dark is simply due to the negative sign in front of the natural logarithm. At 534.4 (Fig. 2C) the exposed 
region is more transmissive, represented by darker features on a lighter background ( $\mathrm{I}_{\text {unexposed }}>$ $\mathrm{I}_{\text {exposed) }}$. Finally, at 537.2 (D), the exposed region in the image is again less transmissive. This type of data should quantitatively provide the change in OD at each energy between the exposed and unexposed regions. Noting that the HSQ can be damaged under soft x-ray exposure, all subsequent data are taken at a single energy, $534.4 \mathrm{eV}$, which is at a contrast maximum in the exposed versus unexposed spectra.

Figure 3A shows raw transmission images of the $1 \mu \mathrm{m} \times 10 \mu \mathrm{m}$ lines at varying latent e-beam exposures probed with STXM at $535.4 \mathrm{eV}$. The features are scanned laterally with a pixel size of 30 $\mathrm{nm} \times 40 \mathrm{~nm}$. At this energy, the features become more transmissive with dose ( $\mathrm{I}_{\text {exposed }}$ is increasing) indicating a progressive change in the local oxygen binding environment with increasing e-beam exposure dose. One dimensional profiles (Fig. 3B), plotted as the absolute value $\mid-$ $\ln \left(\mathrm{I}_{\text {exposed }} / \mathrm{I}_{\text {unexposed }}\right) \mid$, where $\mathrm{I}_{\text {unexposed }}$ is measured $\sim 15$ microns from the features, are obtained by averaging the STXM scan lines at $535.4 \mathrm{eV}$ vertically and plotting along the transverse direction of the pattern. For these features: $\operatorname{Dose}(m C)=0.4 *(1.05)^{\left(\frac{d i s \tan c e}{2}\right)-1}$, i.e. Dose $(\mathrm{mC})=1.2 \mathrm{mC}$ at $50 \mu \mathrm{m}$ along the patterning direction. As expected, the extent of chemical change increases with increasing dose. In the spectra from Fig. 1, this corresponds to the loss of the shoulder of the peak at $535.8 \mathrm{eV}$. This increase in chemical contrast occurs long past the point where the dissolution contrast saturates (i.e. $\sim 1 \mathrm{mC} / \mathrm{cm}^{219}$ ). Particularly significant is the rise in the areas between the lines (henceforth referred to as background reaction) as the line dose increases. Interestingly enough, this background signature is observed at distances greater than $12 \mu \mathrm{m}$ away from the last e-beam exposed feature. In addition, the background between features is measurable even at typical e-beam operational doses of $1000-2000 \mu \mathrm{C} / \mathrm{cm}^{2}$. 
This appearance of chemical contrast in the background is also observed with soft x-ray exposure of unbaked HSQ (spun-on but not thermal treatment). Squares $(0.5 \mu \mathrm{m} x 0.5 \mu \mathrm{m})$ were $\mathrm{x}-$ ray exposed in the STXM with increasing dwell time (dose) and then reimaged at $535.4 \mathrm{eV}$. As shown in Fig. 4, the appearance of chemical contrast is evident outside the regions of the exposed squares, which are drawn in for a visual aid.

To identify the mechanism for the background cross-linking, we consider several factors. Potential causes of the background chemical contrast could be backscattered electrons, heating, or diffusion of chemicals beyond the exposed the reaction front. For these experiments, the HSQ films are exposed on thin silicon nitride membranes. Under these conditions backscatter from $100 \mathrm{keV}$ electrons is negligible. In addition, the background reaction is observed under STXM exposure where there should be no e-beam backscatter effects. Beam heating can also be ruled out because the film is thin. For these films, the temperature rise due to heat deposition from the electron beam is significantly less than one degree (see calculation Appendix I).

We speculate that diffusion of the exposure-initiated radicals and reaction products beyond the exposed region is the most likely cause for the chemical conversion of the HSQ outside the exposed area. Thermal desorption spectroscopy during conventional thermal curing of $\mathrm{HSQ}^{20}$ indicates that the reaction products are water, hydrogen molecules or atoms, and silane or their intermediates. As silane removal occurs at much higher temperatures, $\sim 500{ }^{\circ} \mathrm{C}$, we focus on hydrogen and water here.

There is a strong interplay between hydrogen pretreatments and hydroxyl bond formation in both silsesquioxane and silica materials. Water absorption is a concern with silsesquioxanes used as low-k dielectrics because it raises the dielectric constant by providing a permanent dipole. Typical moisture uptake values in HSQ are give as $2.2 \%$ although the absorption can vary strongly based on porosity. ${ }^{11}$ The curing mechanism is also important as cures that form a greater number of hydrophilic 
$\mathrm{Si}-\mathrm{OH}$ bonds can cause more water uptake. In addition, higher water content results in a lower thermal stability. ${ }^{11}$ Liu and Change, et al. studied the effects of hydrogen treatment on HSQ. ${ }^{21}$ HSQ treated to $\mathrm{H}_{2}$ plasma has reduced dielectric constants and leakage currents presumably because dangling bonds are passivated by $\mathrm{H}$ atoms and cannot react as easily with moisture. This is consistent with studies of optical absorption losses in silica fibers where the presence of $\mathrm{Si}-\mathrm{OH}$ can increase absorption losses after radiation damage while hydrogen gas treatment can mitigate the effects of gamma radiation damage. ${ }^{22}$ Hydrogen atoms have been observed by electron paramagnetic resonance, presumably caged in HSQ polymers under some conditions. ${ }^{23-25}$

Based on these studies, we suspect that water, hydroxyl, and/or hydrogen molecule or hydrogen atom reaction products, formed as a result of e-beam exposure, play a large role in partially chemically reacting the unexposed region. It is possible that some radical or atom products diffuse through the HSQ matrix and propagate the reaction front. However, due to the complexity of the interplay between the products and the polymerization reactions, the exact mechanism remains unclear.

Figure 5 shows a plot of $\left|-\ln \left(\mathrm{I}_{\text {exposed }} / \mathrm{I}_{\text {unexposed }}\right)\right|$ vs. dose for a number of exposed feature sizes and spacings obtained by STXM imaging at $535.4 \mathrm{eV}$. For the identical dose, the change in OD increases with increasing feature size, demonstrating an area-dependent chemical conversion of the ebeam exposed material. The diffusion of "products" could easily give rise to an area-dependent dose because the chemical reactions in areas adjacent to previously exposed area would be expected to change with increasing reaction "product" concentration, which in turn is area dependent. Depending on the diffusion coefficient, pattern area, exposure density, fill direction, current and dose, reactant products can diffuse and chemically alter areas adjacent to the exposed area. Zhao et al. measured a diffusion coefficient of water in HSQ cured at $300^{\circ} \mathrm{C}$ in $\mathrm{N}_{2}$ as $3.61 \times 10^{-10} \mathrm{~cm}^{2} / \mathrm{s}$ at room temperature by a quartz crystal microbalance method. ${ }^{26}$ The diffusion front under these circumstances would be 
too slow to alter the chemical sensitivity prior to e-beam exposure. For example, the time required to write a $1 \mu \mathrm{m} \times 10 \mu \mathrm{m}$ area exposed at $1000 \mathrm{mC} / \mathrm{cm}^{2}$ is $200 \mathrm{~ms}$ while the diffusion length in $200 \mathrm{~ms}$ is only $32 \mathrm{~nm}$. However, we expect the partially cured HSQ, which has a lower density, may allow much faster diffusion. In addition, the diffusion of the relevant species may be significantly different in the e-beam exposed materials than in the samples of Zhao et al. In any case, the measurement of Zhao et al. provides a lower bound on potential diffusion coefficients for water in HSQ.

\section{Atomic Force Microscopy Results}

Atomic Force microscopy measurements of latent e-beam exposed features show a change in HSQ density with dose that is also dependent on the exposed feature size (Fig. 6). This graph is data taken on a $250 \mathrm{~nm}$ thick HSQ film on plain silicon wafers (features are spaced far enough apart to avoid backscatter exposure from neighboring features). Generally, as the dose is increased, the change in feature thickness relative to background first increases at low dose, goes through zero as the dose continues to increase, and then decreases in thickness as the dose is increased further. The smallest features do not achieve the same magnitude of thickness changes observed with the larger features. This may be in part due to mechanical constraints and stress. However, the results may be important in the context of product diffusion playing a role in the chemical conversion of the HSQ. Obviously this deserves careful attention in further studies of the HSQ exposure mechanism.

Not only were these thickness changes observed on Si wafers, but also on HSQ exposed on silicon nitride membranes. On the membrane, the same thickness trends were observed with increasing dose (first a positive thickness change, then through zero, and then a negative thickness change). However, as dose approached $40 \mathrm{mC} / \mathrm{cm}^{2}$, the thickness again started to increase. It is unclear why the trends differed slightly with different substrates but we plan future investigations on HSQ substrate interactions. 
Figure 7 shows AFM images of a $10 \mu \mathrm{m}$ square exposed at $40 \mathrm{mC} / \mathrm{cm}^{2}$ e-beam dose before development (A) and after development (B) in a $2 \%$ solution of tetramethyl ammonium hydroxide (TMAH) in water. Although the dose is high relative to practical exposure doses, the results demonstrate how the effective dose changes are sensitively recorded in the HSQ and how adjacent areas are affected by the dose in neighboring regions.

There are several fine features associated with the $10 \mu \mathrm{m}$ square exposure. There is an increase in thickness, relative to background, outside the $10 \mu \mathrm{m}$ exposed area that extends approximately $1 \mu \mathrm{m}$ (labeled (a) in the line scan under Fig. 7A). Similar to the OD background rise observed with the STXM, this can be indicative of film changes outside the exposed region. In this case, however, it may also be related to mechanical stress.

Also noteworthy are the various thickness changes within the $10 \mu \mathrm{m}$ exposed area itself. The four shapes that construct the overall $10 \mu \mathrm{m}$ square are sub-shapes defined by the exposure algorithm. Each sub-shape is defined by a "spiral in" scan of the e-beam. The " $X$ " that is visible in the middle of the largest sub-shape is an artifact of this spiral in ((b)) in Fig. 7A). Errors in placement as the beam turns the corners results in slightly lower doses that correspond to lower dose relative to background along the "X." In the adjacent rectangular minor fields, this artifact results in a line down the center of the feature ((c) in Fig. 7A). The boundary between sub-shapes is also visible ((d) in Fig. 7) and is indicative of an error in alignment between irradiated shapes. Here the effective dose is less and the thickness is higher than background. Finally, it is important to notice the relative thicknesses of the sub-shapes. There are real thickness differences in the features that translate to the developed samples (Fig. 7B). Based on the measured density changes on silicon, the smaller sub-shapes appear to have less effective dose than the largest sub-shape. However, this is somewhat confused by the additional density changes found for HSQ on the silicon nitride membranes. 


\section{Dissolution Contrast and Optical Density}

Fig. 8 plots the SXTM measurements of $\mid-\ln \left(\mathrm{I}_{\text {exposed }} / \mathrm{I}_{\text {unexposed }} \mid\right.$ of the exposed lines and background between the exposed lines. Insets show AFM images of the same sample developed after STXM imaging. Interestingly enough, the same optical density signal in the exposed lines and background has different heights and thus dissolution rates. The features with 20 mOD (Fig. 8A) resulted in a $159 \mathrm{~nm}$ thick HSQ lines whereas the background between the lines with an $40 \mathrm{mOD}$ (areas between lines with very high exposure dose, Fig. 8B) left material $60 \mathrm{~nm}$ thick. We can conclude that the STXM data, which was taken at only one X-ray energy, does not definitively determine the chemistry responsible for the dissolution contrast. This is not suprising because the STXM images were taken to maximize contrast and not to monitor the change in a specific peak. Future experiments are underway to correlate the full NEXAFS spectra for HSQ samples and with Fourier transform infrared spectroscopy and dissolution data.

\section{Summary}

In this study, we have used STXM and AFM to investigate e-beam and EUV exposure of hydrogen silsesquioxane films. We observed chemical contrast both in the exposed and the unexposed regions implying that the exposure reaction is not spatially contained. Using both the STXM technique and AFM, we obtained results that show area-dependent changes in the exposed films. With STXM, the OD change for larger features was much greater than for smaller features and with AFM, the thickness changes were more significant for larger features than smaller features.

In addition, we observed by STXM that chemical changes with dose continue long after dissolution contrast saturates and that the background doses increase proportionally. Fortunately, AFM studies of developed features show that the background regions between features have higher dissolution rates than exposed features even with the same or lower optical densities (measured at 
$535.4 \mathrm{eV}$ ). This demonstrates that the directly exposed and background regions are chemically different.

From this preliminary study, we speculate that the reactants from the exposure are diffusing and chemically altering neighboring regions. This changes the sensitivity of nearby regions and gives rise to an area dependence of sensitivity. However, this is a difficult and dynamic problem as chemistry and density are changing simultaneously. Exposed feature size, proximity, dose, bias (coding of features different than the desired feature), and arrangement of exposure are all important variables that can affect the concentration of reactants and can result in pattern dependent sensitivity. Small area exposures, low doses and thin films should mitigate the destabilizing effects of diffusing species.

Studies are underway to extract full NEXAFS spectra of the exposed, unexposed, and chemically altered background HSQ material and to correlate this information to the localized bonding environment with FTIR. In addition, we are exploring the addition of reactant scavenging thin films and additives. With this information, we will be on our way to understanding the radiation and thermal chemistry of HSQ and thus be able to exploit the full potential of this inorganic resist material and interlayer dielectric.

\section{Appendix I. Beam Heating During Exposure}

Using the relativistic Bethe law to calculate the heat loss per electron to the bulk per mass-thickness, $\Delta \mathrm{x}$, in Joules $\mathrm{m}^{2} / \mathrm{kg},{ }^{27}$

$$
\frac{\Delta Q}{\Delta x}=\left(\frac{e^{4} N_{a}}{4 \pi \varepsilon_{0}^{2} E_{0} \beta^{2}}\right) \frac{Z}{A} \log \left[\frac{0.5 E_{0} \beta^{2}}{13.5 Z}\right]
$$

Where $\mathrm{E}_{0}$ is the beam energy, $\mathrm{B}$ is the relativistic velocity correction, $\mathrm{Z}$ is atomic number, $\mathrm{A}$ is atomic weight, $\varepsilon_{0}$ is the permitivity of free space, e is the charge on an electron, and $\mathrm{N}_{\mathrm{a}}$ is Avagadro's 
number. For both HSQ and $\mathrm{SiN}$, the average $\mathrm{Z}$ is 10 and $\mathrm{A}$ is $0.02 \mathrm{~kg}$, and $\frac{\Delta Q}{\Delta x}$ is $5 \times 10^{-14} \mathrm{~J}^{*} \mathrm{~m}^{2} / \mathrm{kg}$.

The thin plating base contibute insignificantly to the heat deposition.

The power injected by the beam into the film is

$$
P=\frac{\Delta Q}{\Delta x} \rho t \frac{I}{e}
$$

Where $\mathrm{P}$ is power, $\mathrm{I}$ is the beam current, $\mathrm{t}$ is film thickness, and $\rho$ is film density. As thermal conductivity is much higher in the nitride compared to HSQ ( $30 \mathrm{~W} / \mathrm{mK}$ vs 0.1 to $1 \mathrm{~W} / \mathrm{mK}$ ), we assume a model where the underlying nitride acts as a thermal short to the room-temperature silicon supports. In addition, we assume the power from the beam is deposited into the entire exposed shape in a parallel fashion as opposed to a serial fashion as the beam is scanned (this is the worst case scenario). Assuming radial symmetry, and steady state conditions, the heat flow in the nitride is given by

$$
P=-2 \pi r t_{S i N} \kappa_{S i N} \frac{\partial T}{\partial r}
$$

Where $\kappa_{\mathrm{SiN}}$ is thermal conductivity of the silicon nitride and $\mathrm{T}$ is temperature, Substituting Eqn. 3 into Eqn. 4 and solving for T, the temperature rise $\Delta \mathrm{T}$

$$
\Delta T=\frac{\Delta Q}{\Delta x}\left(\rho_{H S Q} t_{H S Q}+\rho_{S i N} t_{S i N}\right) \frac{I}{e 2 \pi t_{S i N} \kappa_{S i N}} \log \left[\frac{R}{r}\right]
$$

Here, $\mathrm{R}$ is the membrane window radius (the distance to the silicon support heat reservoir) and $\mathrm{r}=\mathrm{r}_{\mathrm{o}}$ at the edge of the beam. Using $\mathrm{I}=500 \mathrm{pA}, \kappa_{\mathrm{SiN}}=30 \mathrm{~W} / \mathrm{mK}, \mathrm{t}_{\mathrm{SiN}}=100 \mathrm{~nm}, \mathrm{t}_{\mathrm{HSQ}}=200 \mathrm{~nm}, \rho_{\mathrm{HSQ}} 2.2$ $\mathrm{g} / \mathrm{cm}^{3}$ (value for $\mathrm{SiO}_{2}$, worst case scenario), and $\rho_{\mathrm{SiN}}=3.29 \mathrm{~g} / \mathrm{cm}^{3}, \mathrm{R} / \mathrm{r}_{\mathrm{o}}=100$ (i.e. 1 micron exposed area and $1 \mathrm{~cm}$ membrane), $\Delta T$ is significantly less than 1 degree. 


\section{Acknowledgements}

Thank you to Aditya Joshi and Yuki Sata for calculating the electron beam temperature rise. The Advanced Light source is supported by the Director, Office of Science, Office of Basic Energy Sciences, of the U.S. Department of Energy under Contract No. DE-AC02-05CH11231. Work at the Molecular Foundry was supported by the Director, Office of Science, Office of Basic Energy Sciences, Division of Materials Sciences and Engineering, of the U.S. Department of Energy under Contract No. DE-AC02-05CH1 1231. SRL acknowledges the National Science Foundation, Division of Materials Research, for support of patterned lithography research. 
Bibliography

H. Namatsu, Y. Takahashi, K. Yamazaki et al., J. Vac. Sci. Technol. B 16 (1), 69 (1998).

F. C. M. J. M. van Delft, Microelectron. Eng 46 (1-4), 369 (1999).

B. E. Maile, W. Henschel, H. Kurz et al., Jpn. J. Appl. Phys., Part 1 39, 6836 (2000).

Deirdre L. Olynick, Bruce D. Harteneck, Eugene Veklerov et al., J. Vac. Sci. Technol. B Vol. 22 (6), 3186 (2004).

K. A. Lister, B. G. Casey, P. S. Dobson et al., Microelectron. Eng 73-74 (1), 319 (2004).

I. Junarsa, M. P. Stoykovich, P. Nealey et al., J. Vac. Sci. Technol. B 23 (1), 138 (2005).

Y. Igaku, S. Matsui, H. Ishigaki et al., Jpn. J. Appl. Phys. 41, 4198 (2002).

K. Nakamatsu, K. Watanabe, K. Tone et al., Jpn. J. Appl. Phys. 43 (6B), 4050 (2004).

F. C. M. J. M. van Delft, J. P. Weterings, A. K. van Langen-Suurling et al., J. Vac. Sci. Technol. B 18 (6), 3419 (2000).

H. Namstsu, Y. Takahashi, K. Yamazaki et al., J. Vac. Sci. Technol. B 16 (1), 69 (1998).

K. Maex, M. R. Baklanov, D. Shamiryan et al., J. Appl. Phys 93 (11), 8783 (2003).

G. Li, L. Wang, H. Ni et al., J. Inorg. Organomet. Polym. Mater. 11 (3), 123 (2001).

F. J. Feher, R. Terroba, R. Jin et al., Polym. Mater. Sci. Eng. 82, 301 (2000).

W. Henschel, Y. M. Georgiev, and H. Kurz, J. Vac. Sci. Technol. B 21 (5), 2018 (2003).

J. A. Liddle, F. Salmassi, P. P. Naulleau et al., J. Vac. Sci. Technol. B 21 (6), 2980 (2003).

L. Muntean, R. Planques, A. L. D. Kilcoyne et al., J. Vac. Sci. Technol. B 23 (4), 1630 (2005).

J. Stohr, NEXAFS Spectroscopy. (Springer Verlig, 1992).

E. H. Anderson, V. Boegli, and L. P. Muray, J. Vac. Sci. Technol. B 13 (6), 2529 (1995).

W. Henschel, Y. M. Georgiev, and H. Kurz, J. Vac. Sci. Technol. B 21 (5), 2018 (2003).

Dow Corning personal communication.

P. T. Liu, T. C. Chang, S. M. Sze et al., Thin Solid Films 332, 345 (1998).

J. Stone, J. Lightwave Technol. 5 (5), 712 (1987).

B. Gross, H. Dilger, R. Scheuermann et al., J. Phys. Chem. A 105, 10012 (2001).

M. Mattori, K. Mogi, Y. Sakai et al., J. Phys. Chem. A 2000, 10868 (2000).

M. Päch and R. Stösser, J. Phys. Chem. A 101, 8360 (1997).

J. H. Zhao, I. Malik, T. Ryan et al., Appl. Phys. Lett 74 (7), 944 (1999).

Ludwig Reimer, Transmission Eelectron Microscopy : Physics of Image Formation and Microanalysis, 4 ed. (Springer, 1997). 
Figure Captions:

Fig. 1. STXM data showing NEXAFS spectra at the oxygen edge for unexposed and exposed HSQ (each normalized to $\mathrm{I}_{0}$ taken from a blank $\mathrm{Si}_{3} \mathrm{~N}_{4}$ window) and the difference spectrum.

Fig. 2. Left side: plot of $\left|-\ln \left(\mathrm{I}_{\text {exposed }} / \mathrm{I}_{\text {unexposed }}\right)\right|$ of STXM images of latent e-beam exposure of $10 \mu \mathrm{m}$ squares in HSQ. Images are obtained at $525 \mathrm{eV}(\mathrm{A}), 534.2 \mathrm{eV}(\mathrm{B}), 535.4 \mathrm{eV}(\mathrm{C})$, and $537.2 \mathrm{eV}(\mathrm{D})$. The highest contrast is observed at 535.4. Right side: the corresponding line profiles from the vertically averaged signal across the $10 \mu \mathrm{m}$ squares.

Fig. 3. Raw STXM images of $1 \times 10 \mu \mathrm{m}$ features as a function of dose (A) and a plot of $\mid-$ $\ln \left(\mathrm{I}_{\text {exposed }} / \mathrm{I}_{\text {unexposed }}\right) \mid(B)$. (For these features: $\operatorname{Dose}(m C)=0.4 *(1.05)^{\left(\frac{\text { distance }}{2}\right)-1}$, i.e. Dose $(\mathrm{mC})$ $=1.2 \mathrm{mC}$ at $50 \mu \mathrm{m})$. There is a significant increase in the background optical density as the feature dose increases. The discontinuity in the graph is where STXM data wasnot taken.

Fig. 4. An overview STXM image after latent soft x-ray exposure of an unbaked HSQ sample with increasing exposure time (noted under squares, in milliseconds). Chemical contrast is observed outside the exposed areas (delineated with white dotted squares).

Fig. 5. Plot of $\left|-\ln \left(\mathrm{I}_{\text {exposed }} / \mathrm{I}_{\text {unexposed }}\right)\right|$ of e-beam exposed features as a function of dose and feature size. Each feature is normalized to the same $\mathrm{I}_{\text {unexposed. }}$ The chemical contrast increases 
with increasing dose as expected but has an additional dependence on the size of feature exposed.

Fig. 6. AFM measurements of latent image feature thicknesses relative to background. The heights change as a function of dose and feature size.

Fig. 7. AFM image of latent (A) and developed (B) $10 \mu \mathrm{m}$ features on silicon nitride. Dose is $40 \mathrm{mC}$. The feature (a) shows that density changes occur outside of the exposed area. The feature(b), an " $\mathrm{X}$ " is an artifact of how the e-beam is raster scanned. Features (c) and (d) are explained in the text. As the beam spirals from outside to inside, errors in placement result in lower dose as the beam passes the corners and center of the fill pattern.

Fig. 8. Plot of $\left|-\ln \left(\mathrm{I}_{\text {exposed }} / \mathrm{I}_{\text {unexposed }}\right)\right|$ for the $150 \mathrm{~nm} \times 1 \mu \mathrm{m}$ features and the background between the features. AFM images (Insets A and B) are taken after development. Lines with a lower mOD compared to background areas (areas between the lines exposed at much higher doses) leave thicker developed HSQ features. 


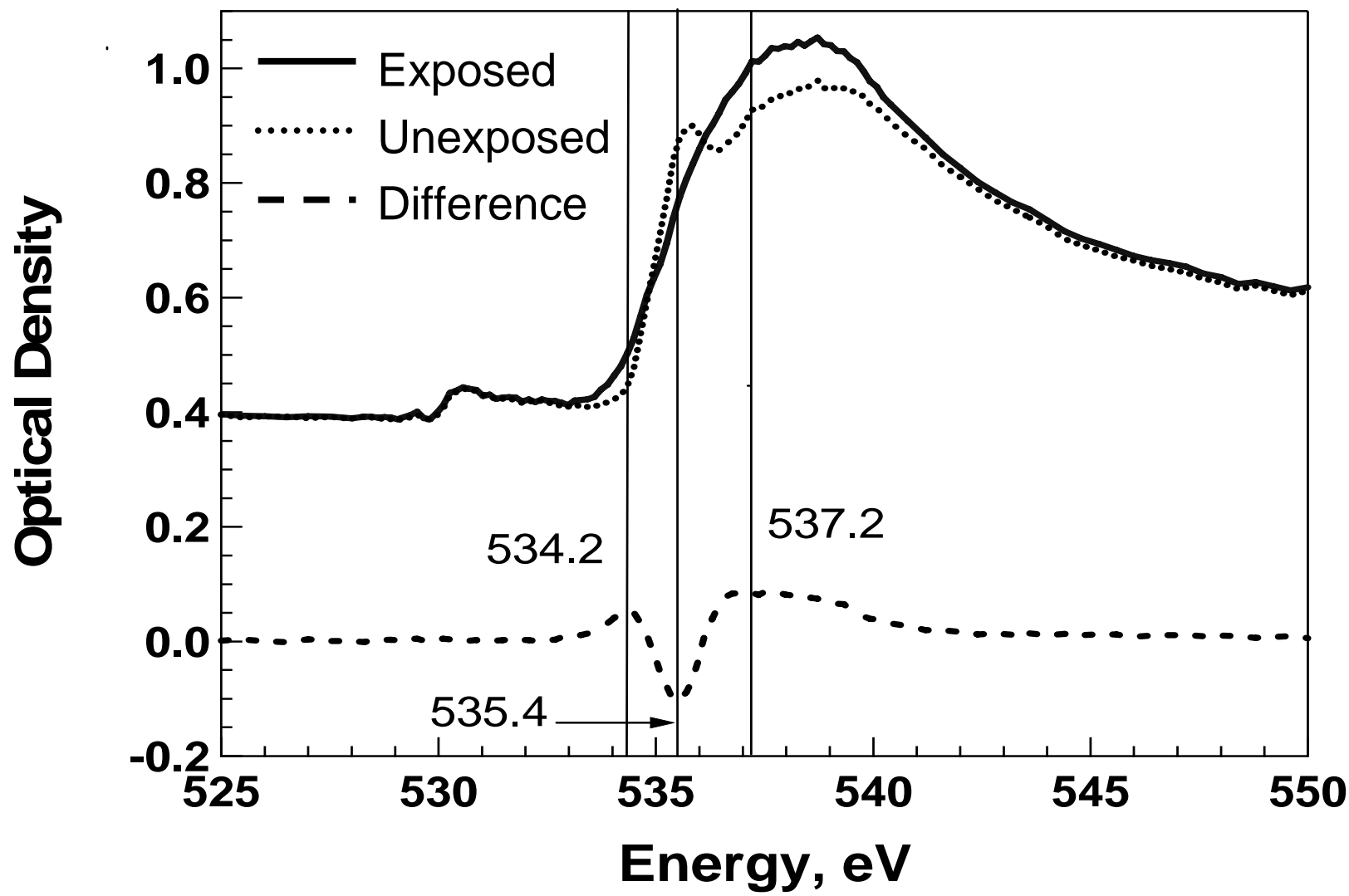

Fig. 1. 


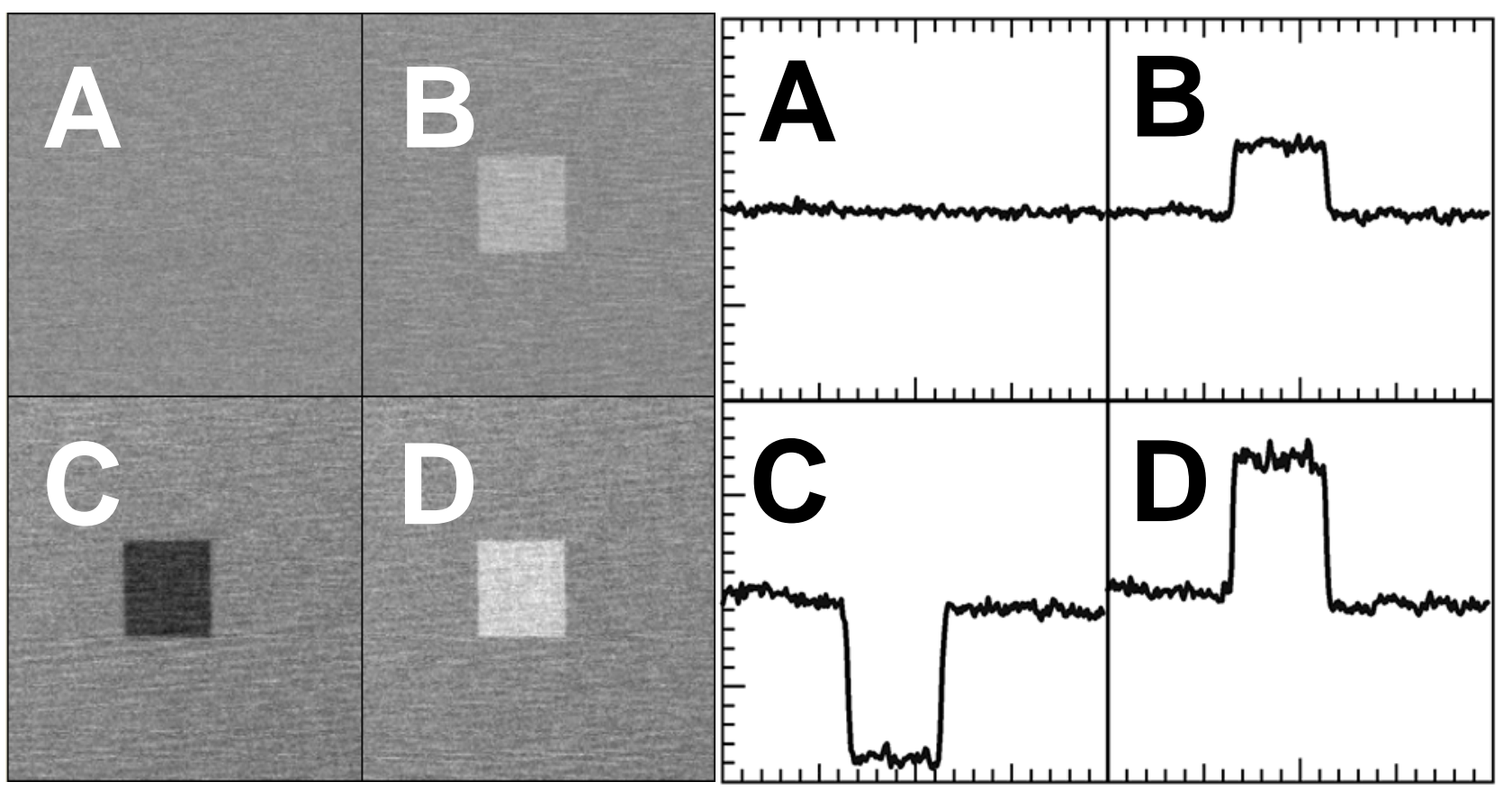

Fig. 2. 


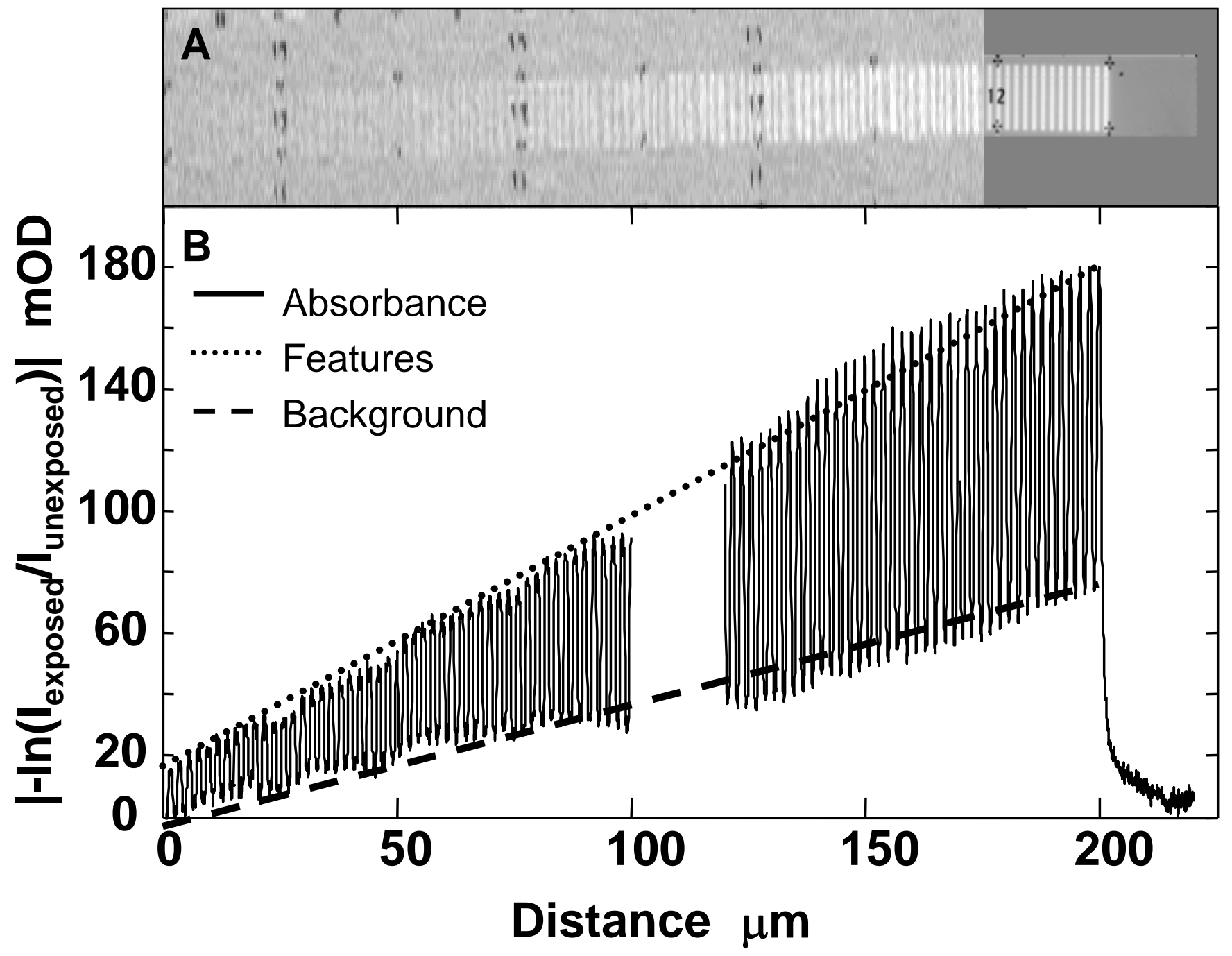

Fig 3. 


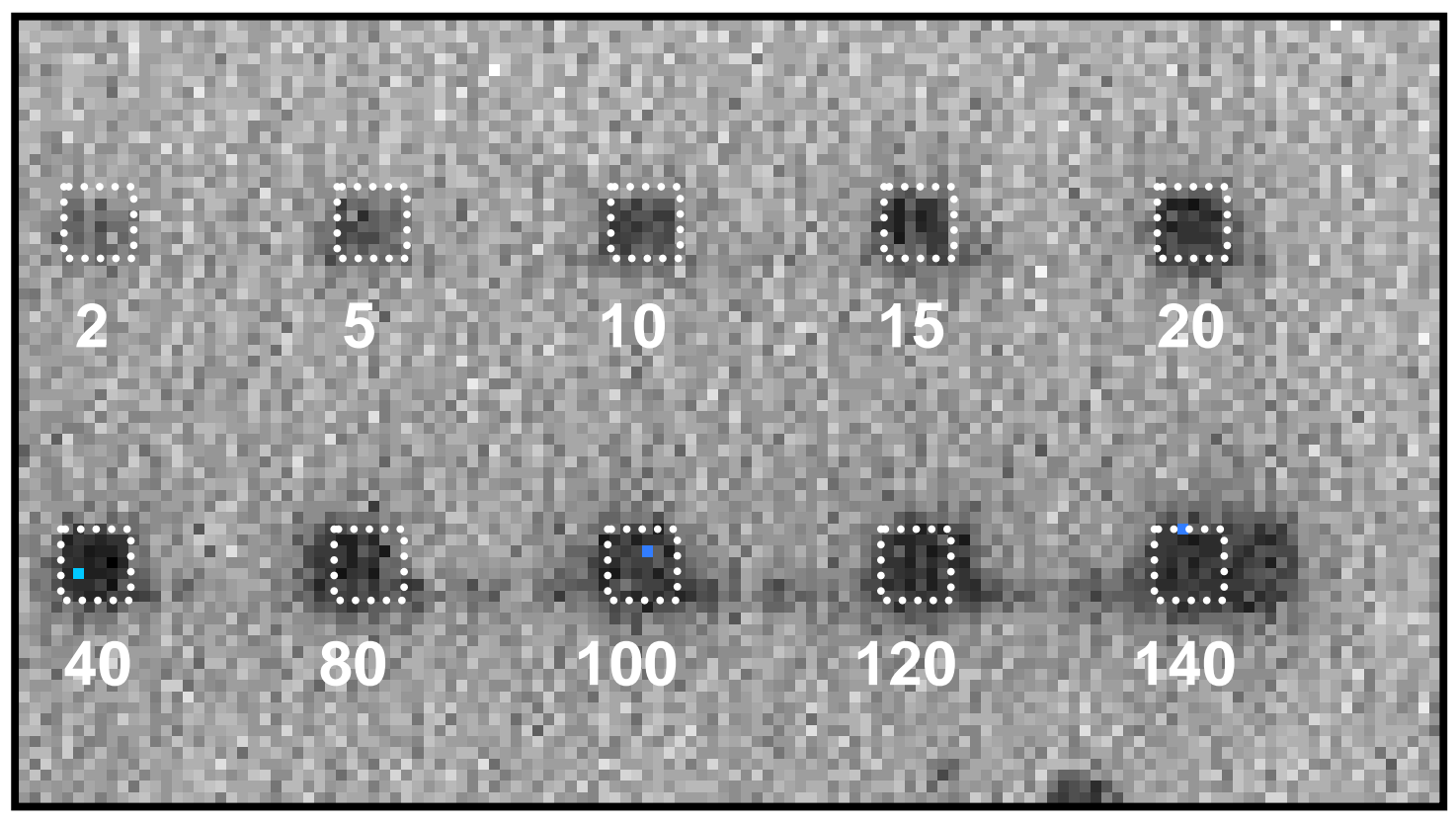

Fig 4. 


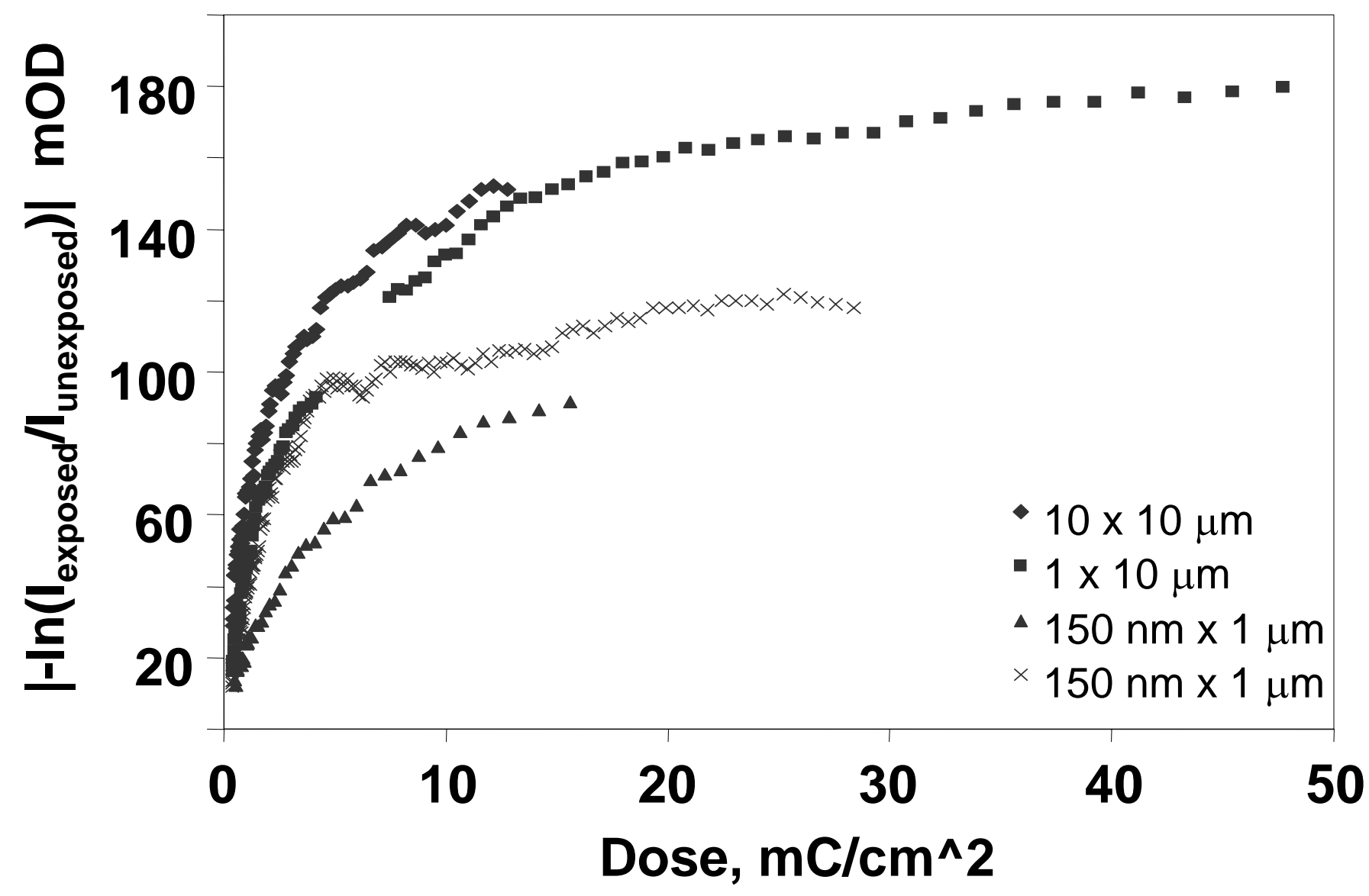

Fig 5. 


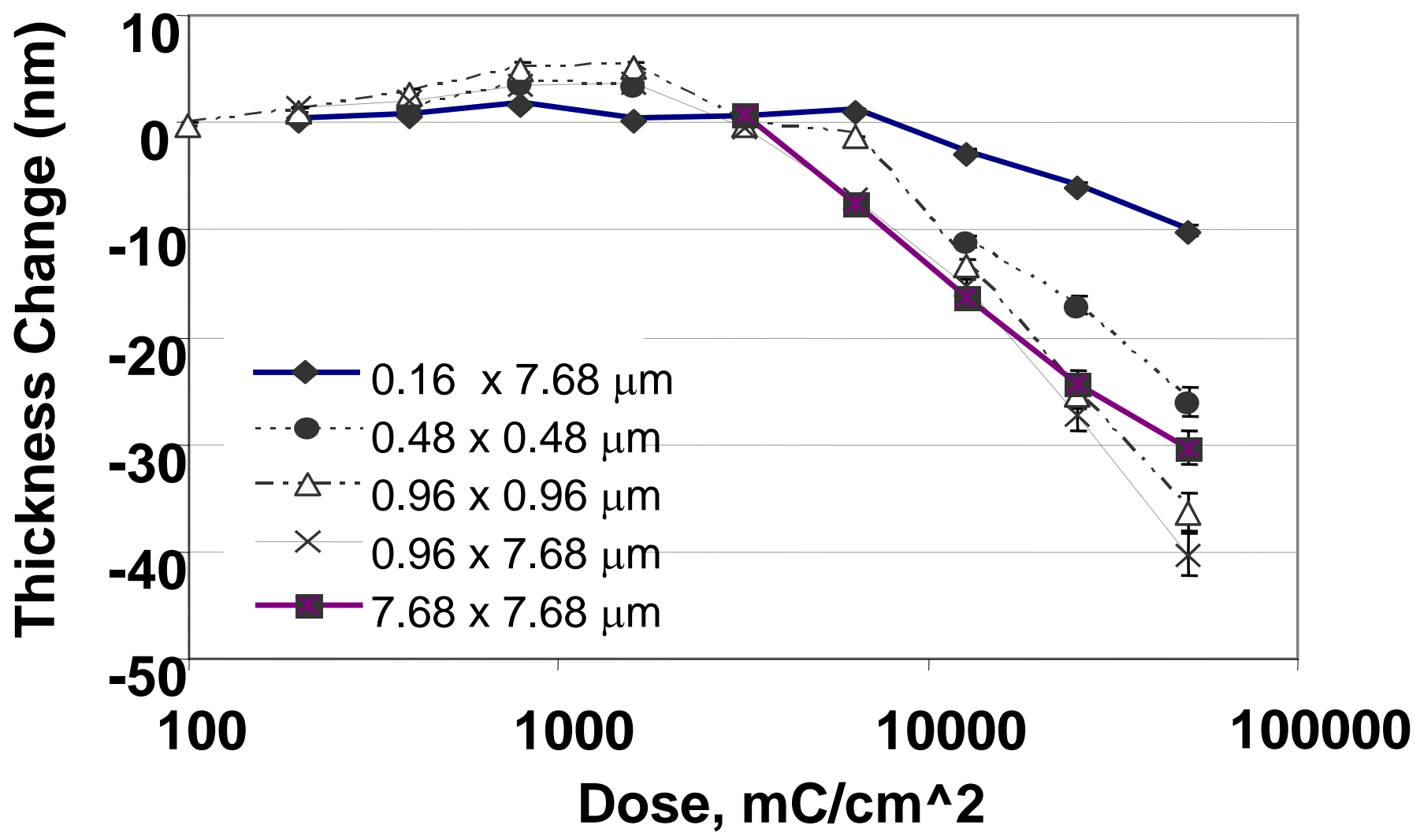

Fig 6. 

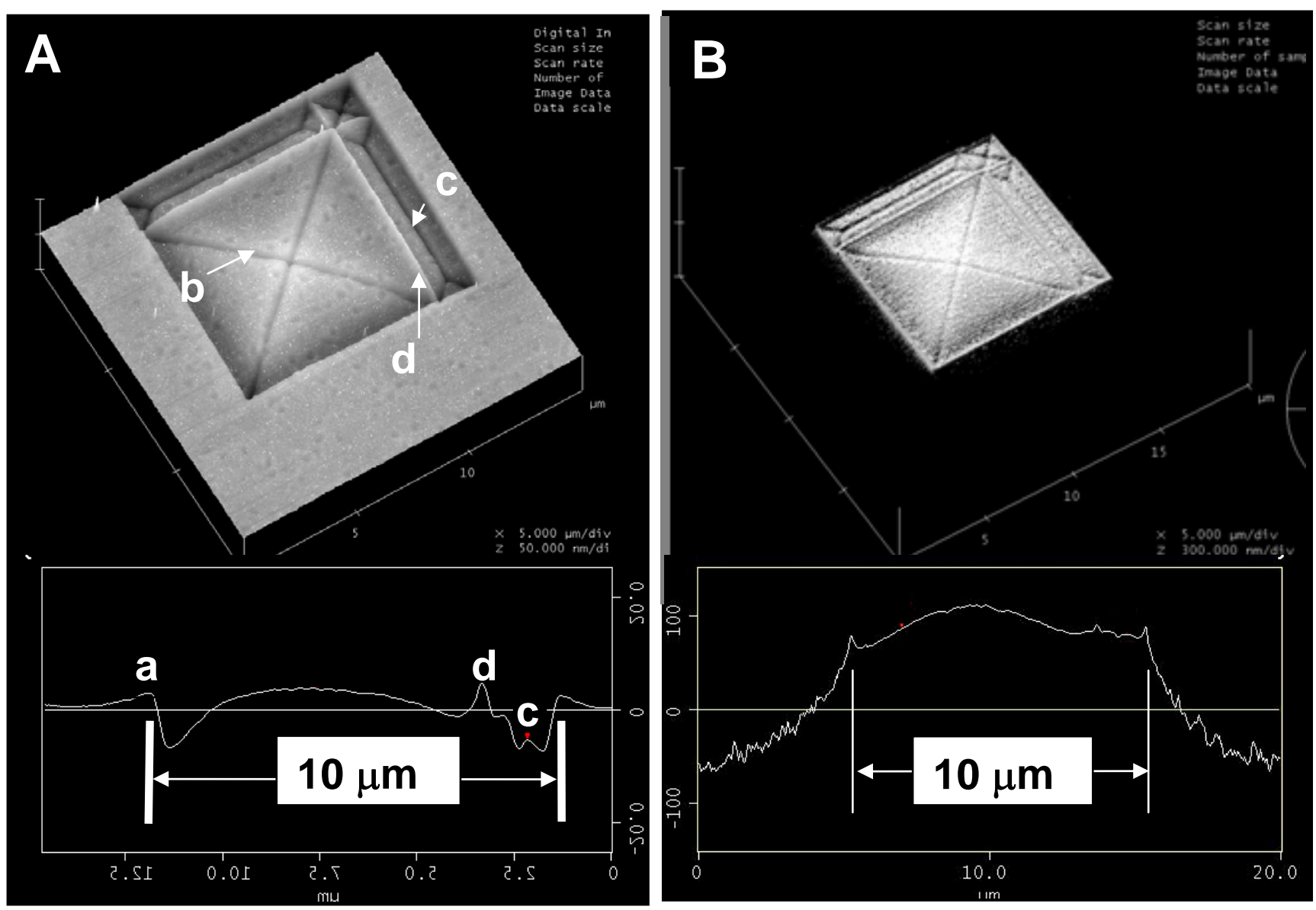

Fig 7. 


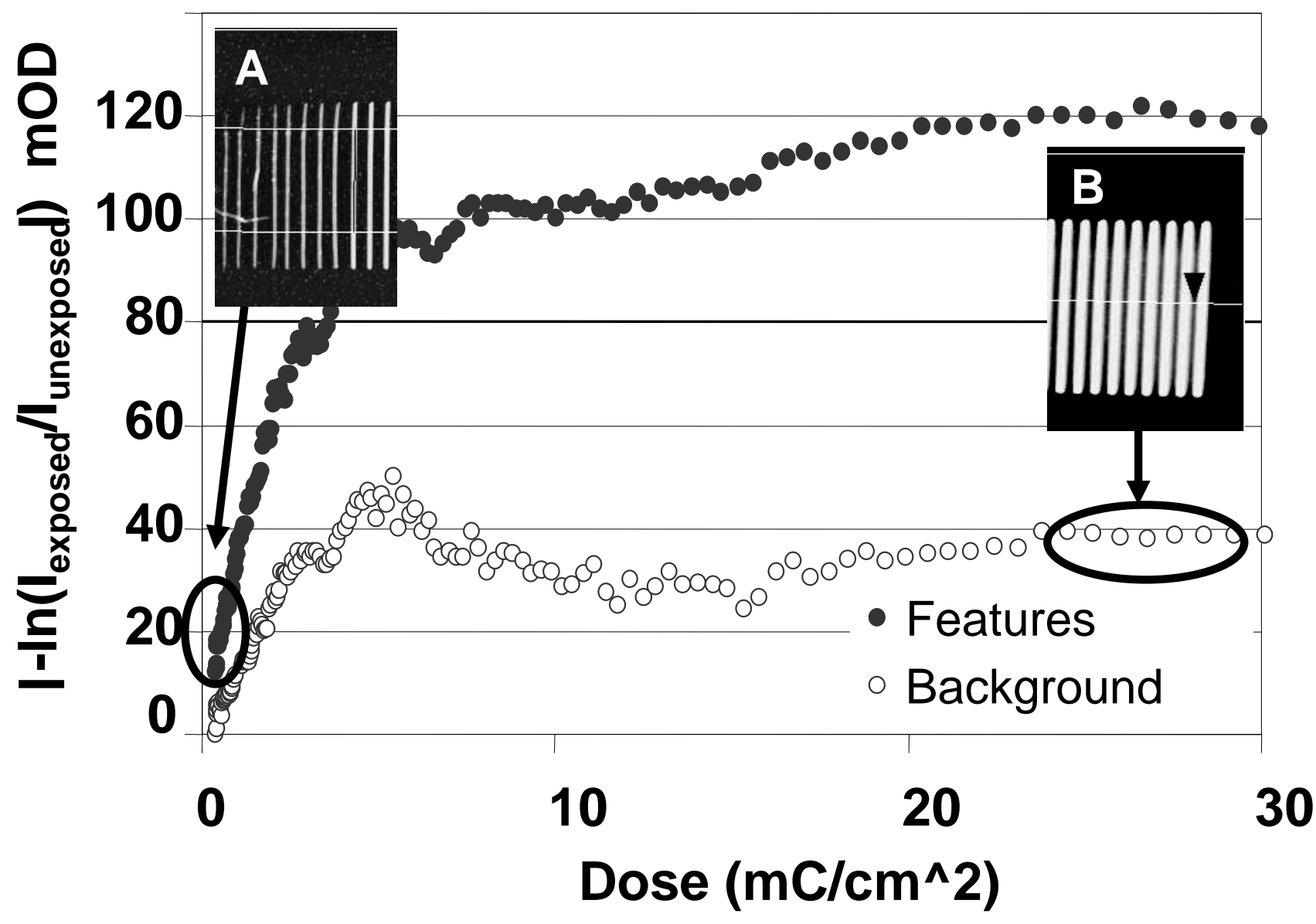

Fig 8. 